\title{
Multi-Targeted Antiangiogenic Tyrosine Kinase Inhibitors in Advanced Non-Small Cell Lung Cancer: Meta-Analyses of 20 Randomized Controlled Trials and Subgroup Analyses
}

\author{
Wenhua Liang', Xuan Wu', Shaodong Hong', Yaxiong Zhang, Shiyang Kang, Wenfeng Fang, Tao Qin, \\ Yan Huang, Hongyun Zhao, Li Zhang*
}

Sun Yat-sen University Cancer Center, State Key Laboratory of Oncology in South China, Collaborative Innovation Center for Cancer Medicine, Guangzhou, China

\begin{abstract}
Background: Multi-targeted antiangiogenic tyrosine kinase inhibitors (MATKIs) have been studied in many randomized controlled trials (RCTs) for treatment of advanced non-small cell lung cancer (NSCLC). We seek to summarize the most up-todate evidences and perform a timely meta-analysis.

Methods: Electronic databases were searched for eligible studies. We defined the experimental arm as MATKI-containing group and the control arm as MATKI-free group. The extracted data on objective response rates (ORR), disease control rates (DCR), progression-free survival (PFS) and overall survival (OS) were pooled. Subgroup and sensitivity analyses were conducted.

Results: Twenty phase II/III RCTs that involved a total of 10834 participants were included. Overall, MATKI-containing group was associated with significant superior ORR (OR $1.29,95 \% \mathrm{Cl} 1.08$ to $1.55, P=0.006$ ) and prolonged PFS (HR $0.83,0.78$ to $0.90, P=0.005)$ compared to the MATKI-free group. However, no significant improvements in DCR (OR 1.08, 1.00 to 1.17 , $P=0.054)$ or OS (HR $0.97,0.93$ to $1.01, P=0.106$ ) were observed. Subgroup analyses showed that the benefits were predominantly presented in pooled results of studies enrolling previously-treated patients, studies not limiting to enroll non-squamous NSCLC, and studies using MATKIs in combination with the control regimens as experimental therapies.

Conclusions: This up-to-date meta-analysis showed that MATKls did increase ORR and prolong PFS, with no significant improvement in DCR and OS. The advantages of MATKIs were most prominent in patients who received a MATKI in combination with standard treatments and in patients who had previously experienced chemotherapy. We suggest further discussion as to the inclusion criteria of future studies on MATKls regarding histology.
\end{abstract}

Citation: Liang W, Wu X, Hong S, Zhang Y, Kang S, et al. (2014) Multi-Targeted Antiangiogenic Tyrosine Kinase Inhibitors in Advanced Non-Small Cell Lung Cancer: Meta-Analyses of 20 Randomized Controlled Trials and Subgroup Analyses. PLoS ONE 9(10): e109757. doi:10.1371/journal.pone.0109757

Editor: William B. Coleman, University of North Carolina School of Medicine, United States of America

Received April 17, 2014; Accepted September 8, 2014; Published October 16, 2014

Copyright: ( $) 2014$ Liang et al. This is an open-access article distributed under the terms of the Creative Commons Attribution License, which permits unrestricted use, distribution, and reproduction in any medium, provided the original author and source are credited.

Data Availability: The authors confirm that all data underlying the findings are fully available without restriction. All data are available from PubMed (http:// www.ncbi.nlm.nih.gov/pubmed/).

Funding: This study was funded by the National High Technology Research and Development Program of China Molecular classification and individualized diagnosis and treatment of lung cancer (grant numbers: 2012AA02A502) and the role of monitoring circulating tumor cells and drug-resistance-associated molecular in the therapeutic prediction, evaluation and mechanism of drug-resistance in EGFR mutant advanced NSCLC patients receiving EGFR-TKI (grant numbers: Wu Jieping Funds 320.6750 .1316$)$. The funders had no role in study design, data collection and analysis, decision to publish, or preparation of the manuscript.

Competing Interests: The authors have declared that no competing interests exist.

*Email: zhangli6@mail.sysu.edu.cn

9 These authors contributed equally to this work.

\section{Introduction}

Lung cancer is the leading cause of cancer-related mortality worldwide, with about $85 \%$ patients diagnosed with non-small cell lung cancer (NSCLC) [1]. Locally advanced or metastatic NSCLC accounts for $80 \%$ patients; for these patients the standard care is systemic chemotherapy [2]. Regardless of the emergence of new agents, however, chemotherapy provides only marginal benefit in overall survival [3].
Another treatment option is to inhibit angiogenesis, a complicated process that is regulated by cellular cues, multiple receptormediated signaling networks, and a number of pro- and antiangiogenic factors [4,5]. Antiangiogenic therapy is designed to minimize the acquisition of nutrients and oxygen diffusion to starve tumors. Vascular endothelial growth factor (VEGF) is a key mediator of angiogenesis which has been well studied. Currently, the only antiangiogenic agent approved for patients with NSCLC is bevacizumab, an anti-VEGF monoclonal antibody [6]. How- 
ever, many other antiangiogenic agents are under clinical development.

VEGF receptor (VEGFR) also plays an important role in the pathways regarding angiogenesis. Multi-targeted antiangiogenic tyrosine kinase inhibitors (MATKIs) are novel agents that target VEGFR-dependent tumor angiogenesis and simultaneously inhibit some other key pathways, such as platelet-derived growth factor (PDGF), fibroblast growth factor (FGF), epidermal growth factor and their associate receptors. Previous studies showed that these small-molecule inhibitors are activitive in a wide variety of cancers [7]. MATKIs could fill in a unique niche for cancer therapeutics, especially in western countries where a relatively small population is suitable for receiving targeted therapies that direct known gene alterations [8]. Recently, these similar MATKIs have showed promising advantages in the treatment of advanced NSCLC [9]. A previous meta-analysis suggested that a regimen of chemotherapy in combination with MATKIs have specific advantages over chemotherapy alone in terms of PFS and ORR, but not in OS [10]. However, it involved only six randomized controlled trials (RCTs) and three agents. Since then, plenty of novel results from phase II/III RCTs have been reported. Thus, we sought to perform a timely meta-analysis to summarize all the evidence including the updated reports. In addition, the abundant data allowed us to carry out some subgroup analyses.

\section{Methods}

\section{Search Strategy}

PubMed, EMBASE, the Cochrane Library as well as the ASCO and ESMO databases from Jan 2005 to Jan 2014 were searched for eligible trials. Search terms were the combination of "nonsmall-cell lung cancer" with any of the following: "multitargeted antiangiogenesis tyrosine kinase inhibitors" or "sorafenib", "sunitinib", "cediranib", "vandetanib", "motesanib", "nintedanib", "pazopanib" or "axitinib". The reference lists of the included studies and recent reviews were checked manually as a supplement. No language restriction was applied.

\section{Eligibility Criteria}

In order for a study to be included in this analysis, the following criteria should be met: 1) phase II or III RCT; 2) studies that compared at least one MATKI-containing regimen to MATKI-free regimens as any line treatments in patients with advanced NSCLC; 3) studies reporting at least one response or survival endpoints. In cases of overlap reports, we included only the latest results. Trials will be excluded if they were not in accordance with the eligible criteria.

\section{Endpoints}

The major endpoints for this meta-analysis were overall survival (OS; defined as the time of randomization to the time of death), progression free survival (PFS; defined as the time of randomization to the time of disease progression or death), objective response rate (ORR, percent of patients whose best response was complete response or partial response according to the Recist 1.1 criteria) and disease control rate (DCR, percentage of patients whose best response was complete response, partial response or stable disease according to Recist 1.1 criteria), as well as adverse events (AEs).

\section{Quality Assessment and Data Extraction}

The quality of each eligible study was rated according to the JADAD score [11]. Baseline clinical characteristics, total number of enrolled patients, number of patients who showed complete response, partial response or stable disease, hazard ratio (HR) of median OS and PFS were extracted by two investigators independently. Discrepancies were discussed by the two investigators to reach consensus. In case of missing data, we contacted the primary investigators through emails.

\section{Statistical Analysis}

We defined the experimental arm as MATKI-containing group and the control arm as MATKI-free group. Pooled hazard ratios (HRs) for survival outcomes (PFS and OS) and pooled odds ratio (ORs) for dichotomous data (ORR, DCR and toxicities) with 95\% confidence intervals (CI) were calculated using the Inverse Variance algorithm and Mantel-Haenszel algorithm. Heterogeneity across studies was assessed with a forest plot and the inconsistency statistic $\left(\mathrm{I}^{2}\right)$. An $\mathrm{I}^{2}$ of $25 \%, 50 \%$ and $75 \%$ was considered the cutoff of mild, moderate and severe statistical heterogeneity respectively. A random-effects model was employed in case of the existence of potential heterogeneity; however, both fixed-effect and random effects model were tested. All calculations were performed using STATA 11.0 (StataA Corp, College Station, TX). Subgroup analysis was conducted according to the line of treatment $\left(1^{\text {st }}\right.$-line vs. $2^{\text {nd }}-/ 3^{\text {rd }}$-line/maintenance $)$, involved histological type (unselective population vs. selective population for non-squamous carcinoma), the comparison pattern (MATKI + standard treatment/standard treatment vs. MATKI/standard treatment) respectively. Interaction tests were conducted to assess the inter-subgroup differences. Sensitivity analyses were performed. Graphical funnel plots were generated to visually inspect for publication bias. The statistical methods for detecting funnel plot asymmetry were the rank correlation test of Begg and Mazumdar and the regression asymmetry test of Egger [12,13]. For all analyses, $\mathrm{P}<0.05$ was considered statistically significant.

\section{Results}

\section{Selection and Features of Included Studies}

Six agents (vandetanib, sunitinib, cediranib, sorafenib, motesanib and nintedanib) could be analyzed while trial data for the other agents remain immature. After screening, 20 phase II/III RCTs ( 7 for vandetanib, 2 for sunitinib, 3 for cediranib, 5 for sorafenib, 2 for motesanib, and 1 for nintedanib) involving a total of 10834 participants [14-33]. Figure 1 showed the flow chart of study selection. The definitions of all studied endpoints were consistent among included studies. Among all studies, 10 trials enrolling patients who previously received chemotherapy while the other 10 included chemo-naïve patients. In addition, five studies limited the eligible histological type to non-squamous carcinoma [18,20,31-33]. The 2007 study by Heymach et al. [24] contained two dose groups $(100 \mathrm{mg} / \mathrm{d}$ and $300 \mathrm{mg} / \mathrm{d})$ and the 2008 study by Heymach et al. [27] contained two experimental regimens, they were therefore divided into two separate arms for all analyses. Similarly, Blumenschein's study [32] was divided into two arms: motesanib $125 \mathrm{mg}$ arm and motesanib $75 \mathrm{mg}$ arm respectively. Table 1 summarized the characteristics of both the included agents and studies.

\section{Meta-analysis of the MATKI-containing Regimens versus MATKI-free Regimens}

When compared to MATKI-free group, MATKI-containing group was associated with significantly superior ORR (OR 1.29, $95 \%$ CI 1.08 to $1.55, P=0.006$; Fig. 2A) and longer PFS (HR $0.83,95 \%$ CI 0.78 to $0.90, P=0.005$; Fig. 2 C). However, no significant improvement in DCR (OR 1.08, 95\% CI 1.00 to 1.17 , $P=0.054$; Fig. 2B) and OS (HR 0.97, 95\% CI 0.93 to 1.01 , 


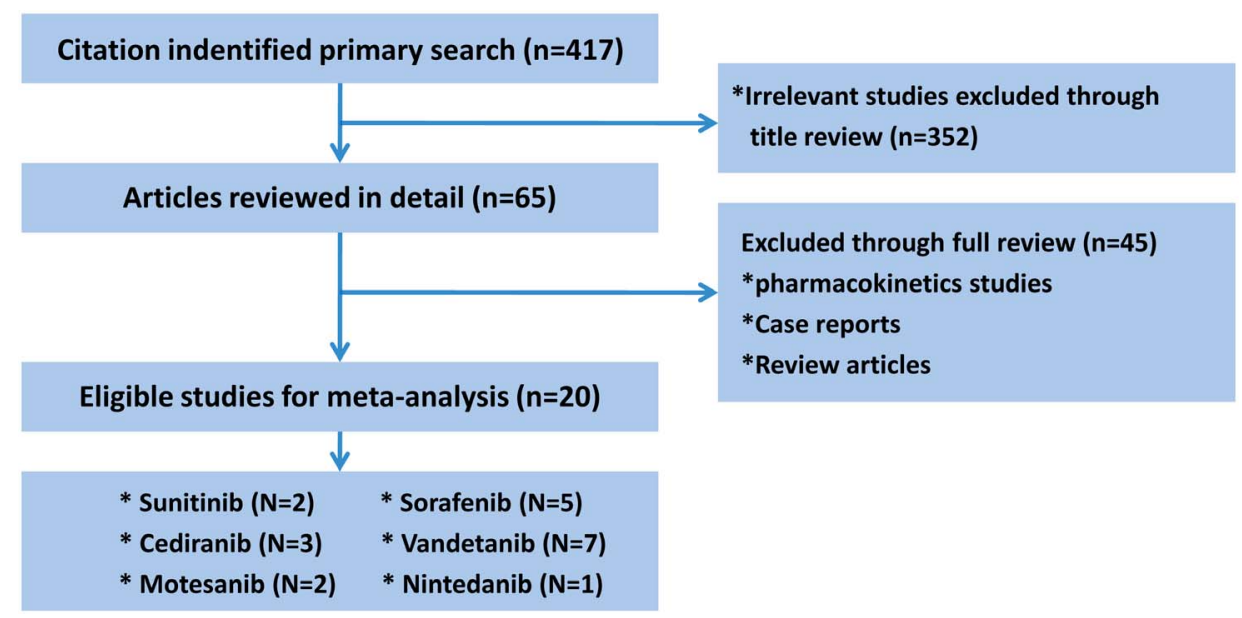

Figure 1. Flow chart of study selection. doi:10.1371/journal.pone.0109757.g001

$\mathrm{P}=0.106$; Fig. 2D) was observed. Figure 2 illustrates all results of the overall meta-analyses.

\section{Subgroup Analyses, Sensitivity Analyses and Publication Bias}

When stratifying patients according to chemotherapy history, we observed that greater benefits of MATKIs in ORR and DCR were presented in patients who failed the prior chemotherapy than those without any prior chemotherapy regarding all outcomes $\left(2^{\text {nd }}-/ 3^{\text {rd }}-\right.$ line $/$ maintenance vs. $1^{\text {st }}-$ line: ORR, OR 1.547 vs. 1.116 , $\mathrm{P}_{\text {interaction }}=0.08 ; \mathrm{DCR}, \mathrm{OR} 1.183$ vs. 0.956, $\mathrm{P}_{\text {interaction }}=0.003$; PFS, HR 0.817 vs. $0.848, \mathrm{P}_{\text {interaction }}=0.60$; OS, HR 0.965 vs. $0.97, \mathrm{P}_{\text {interaction }}=0.92$ ) (greater $\mathrm{OR}$ indicated better ORR or DCR, while smaller HR indicated better PFS or OS). In terms of histology, the pooled results of studies that includes tumors of all histological types (not limited to non-squamous NSCLG) showed a favorable trend in comparison with studies selectively enrolled non-squamous carcinoma especially in ORR and PFS (unselective population vs. selective population: ORR, OR 1.375 vs. 1.140, $\mathrm{P}_{\text {interaction }}=0.33 ; \mathrm{DCR}, \mathrm{OR} 1.077$ vs. $1.081, \mathrm{P}_{\text {interaction }}=0.97$; PFS, HR 0.811 vs. 0.899, $\mathrm{P}_{\text {interaction }}=0.21$; OS, HR 0.965 vs. $\left.0.966, P_{\text {interaction }}=0.98\right)$. We found that the studies whose experimental arms investigated regimens of adding MATKIs to the regimens in the control arm were associated with greater benefits on PFS (HR 0.798 vs. 0.998, $P_{\text {interaction }}=0.03$ ). In addition, significance of DCR improvement (OR 1.095, 95\% CI 1.016 to $1.180, P=0.018$ ) and OS benefits (HR 0.951, 95\% CI 0.907 to $0.998, \mathrm{P}=0.042$ ) were found to be statistically significant, which differed from the overall results and the results of other subgroups. Conversely, we failed to observe any benefit from studies comparing the efficacy of MATKI to the standard regimens in the control arms. All results of the subgroup analyses (including test of interaction) are illustrated in Figure 3 and were summarized in Table S1.

We conducted a sensitivity analysis by using a fixed-effects model (Table S1). We also conducted a sensitivity analysis to examine the pooled results after excluding one study by lee et al. [30] because it compared vindetanib to placebo which differed significantly from all other included trials. The conclusions regarding all outcomes were not altered in sensitivity analyses. In regard to the publication bias, no significant bias was observed in analyses of all endpoints through both Begg's test and Egger's test $(\mathrm{P}>0.05)$.

\section{Discussions}

The angiogenesis pathways, which play a critical role in the nourishment of both the tumors and metastatic lesions, have been targeted as a therapeutic option. Bevacizumab, a monoclonal antibody that binds to VEGF, is the only antiangiogenic drug approved for the treatment of NSCLC since it showed overall survival improvement when combined with chemotherapy [6]. An alternative approach is to shut down the VEGFR functions. Therefore, several multi-targeted tyrosine kinase inhibitors (MATKIs) that target VEGFR as well as other key pathways concerning angiogenesis and tumor proliferation are being developed. In some solid tumors, such as renal cell carcinoma and hepatocellular carcinoma, MAKTIs did reduce tumor burden and prolong overall survival [7]. Several of these agents have been evaluated in a series of phase II/III clinical trials for NSCLC patients. A previous meta-analysis suggested that regimens of chemotherapy plus MATKIs were superior to chemotherapy alone in terms of PFS and ORR, but not in OS [10]. However, this meta-analysis involved only six RCTs and three agents (sorafenib, sunitinib, and cediranib). There have been more than ten novel studies published afterwards. Thus, we believed it necessary to update the results using the new evidence and explore some novel information.

According to the current results, regimens containing sorafenib, sunitinib, cediranib, vandetanib, motesanib or nintedanib had substantial improvements for ORR and PFS outcomes, when compared with regimens free of these agents. In contrast, benefits in DCR and OS were not statistically significant. Since ORR is a part of DCR, we speculated that the underlying reason for a difference in associated benefits between ORR and DCR was that MATKIs failed to deliver any additional benefit in patients that presented primary resistance to standard treatments (chemotherapies or TKIs). This hypothesis was strengthened by our findings in the subgroup analyses: MATKIs failed to achieve any improvement when using alone. The current results could be explained by a widely accepted hypothesis that anti-angiogenic agents can transiently normalize the abnormal structure and function of tumor vessels to make it more efficient for drug delivery [34]. Therefore, MATKIs could 'rescue' those who actually 

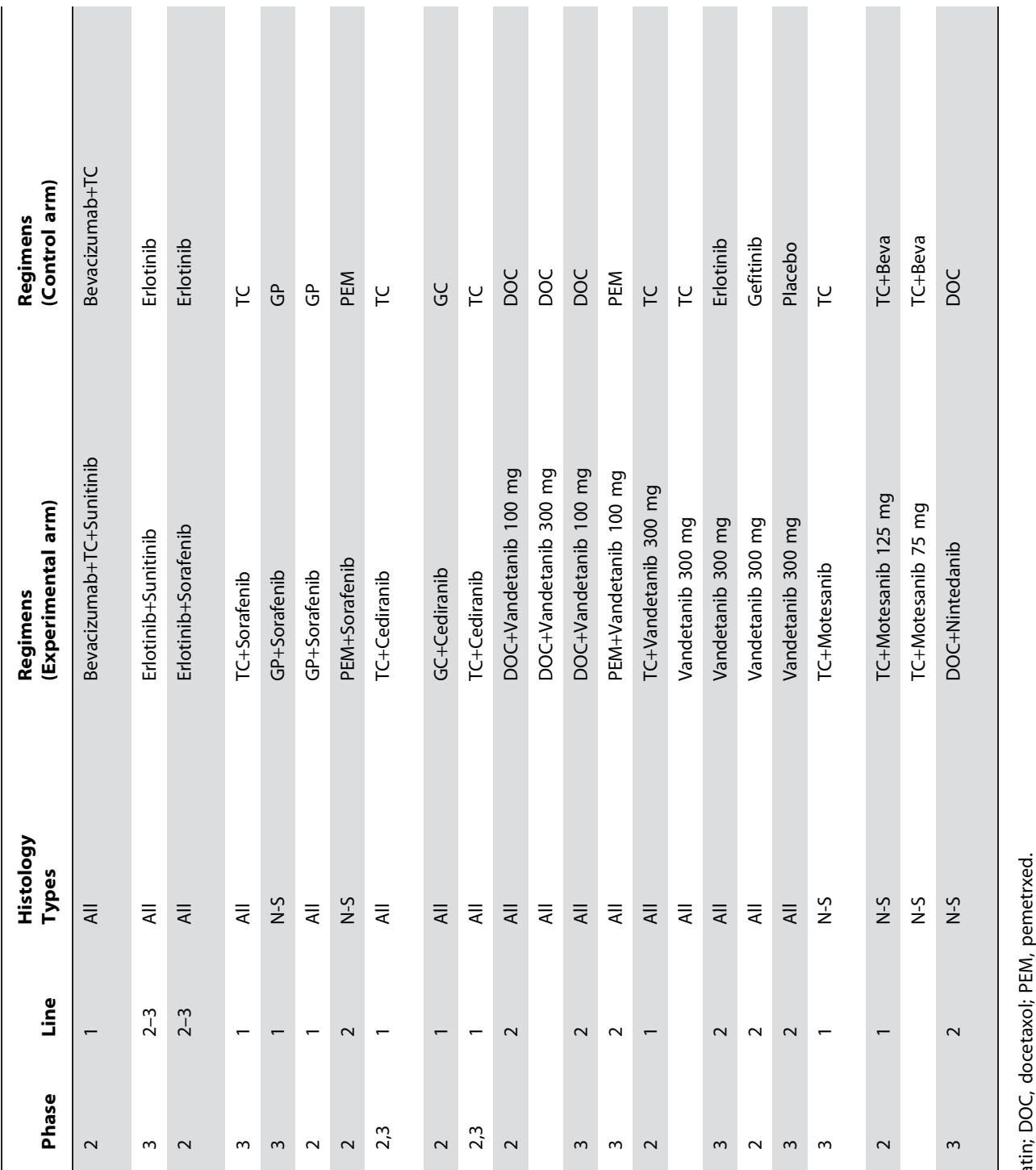

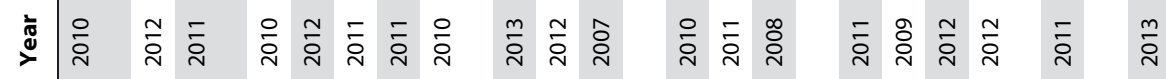

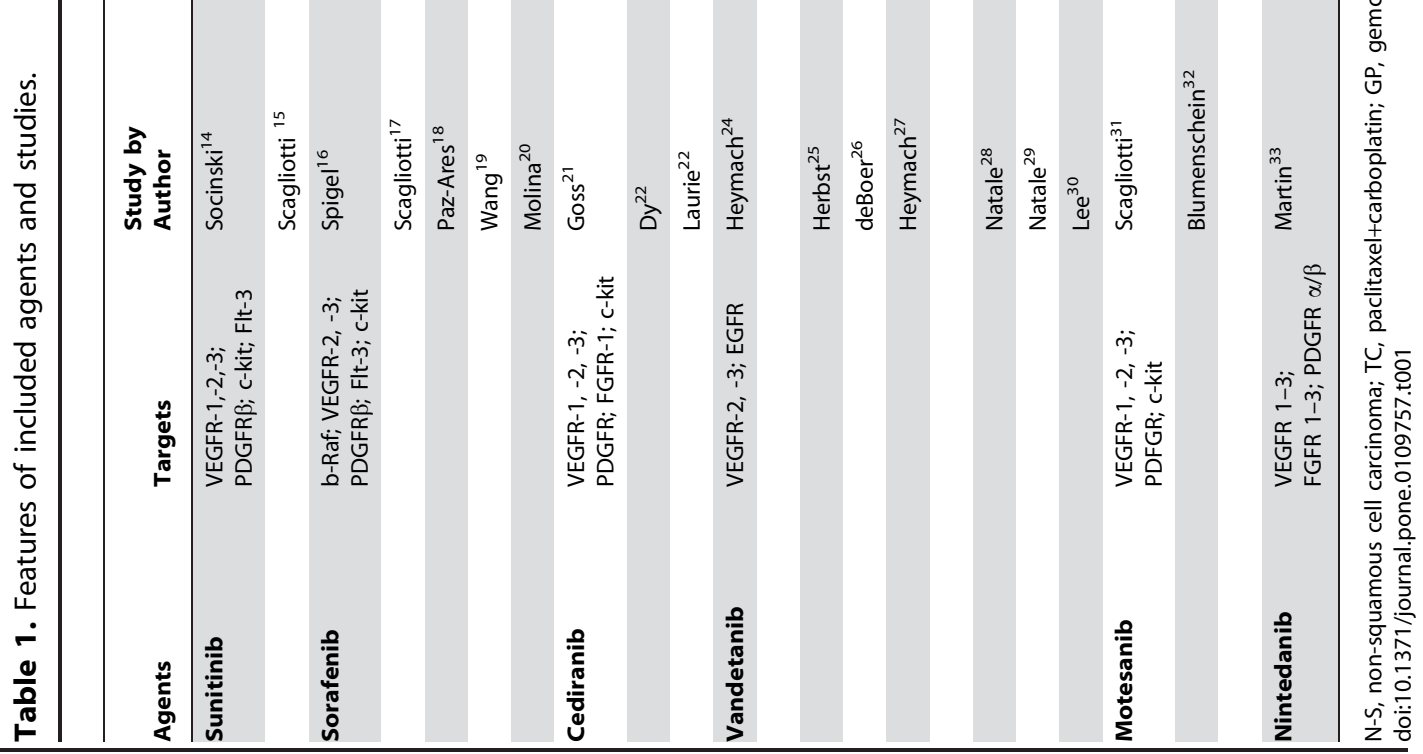




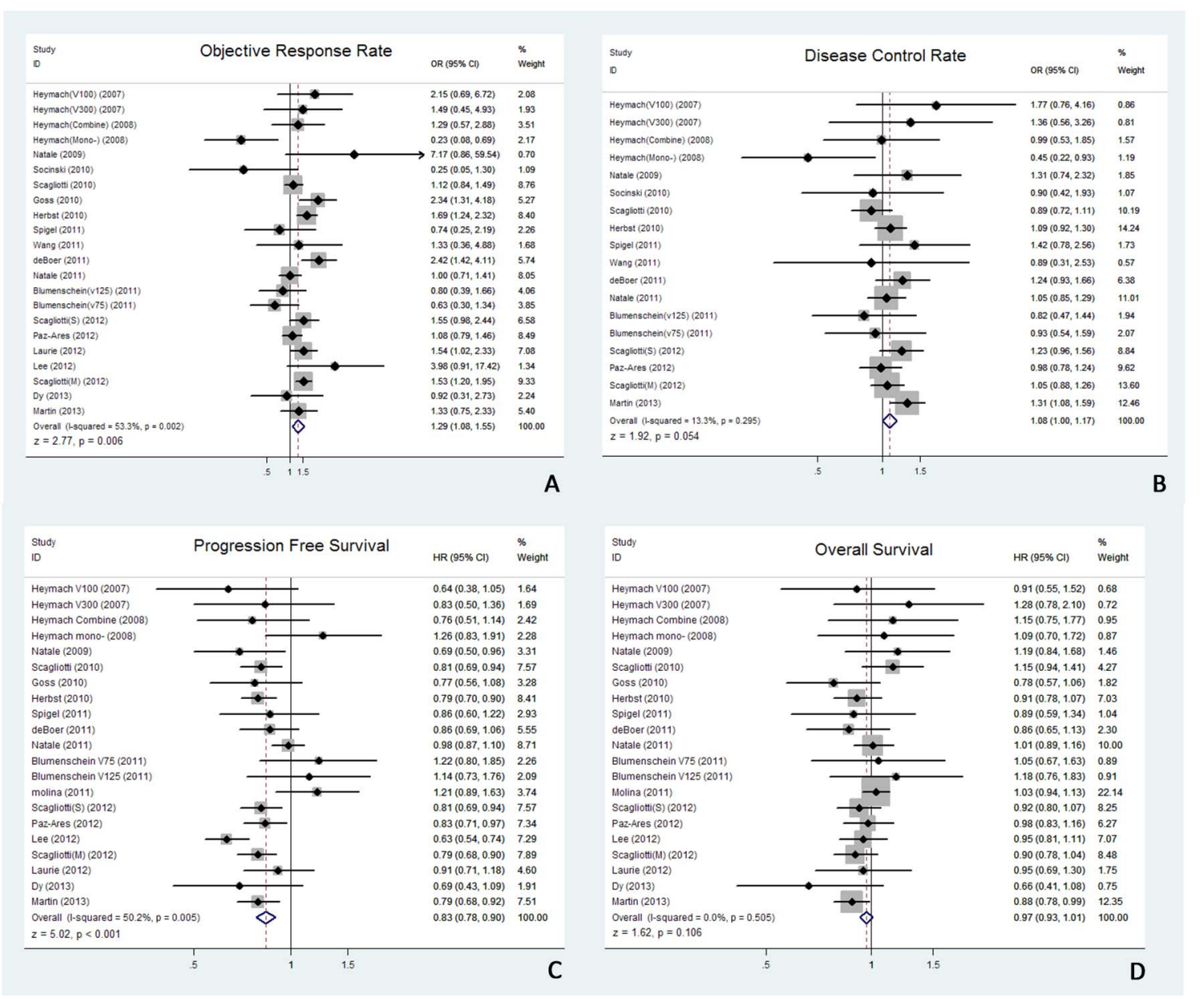

Figure 2. Meta-analyses of MATKIs-containing regimens versus MATKIs-free regimens. $A, O R R ; B, D C R ; C, P F S ; D, O S$. doi:10.1371/journal.pone.0109757.g002

respond to chemotherapy but suffered from drug accessibility; this finally translated into significantly improved pooled ORR; however patients who were non-responsive remained the same status, therefore there was no significant increase in DCR. Another hypothesis derived from these results was that regulating the VEGFR pathway might play a more important role than inhibition of other targets (EGFR, PDGFR, etc) in the use of MATKIs when no selection for specific patients was conducted. It implied that MATKIs might have to work in combination with other anti-proliferative agents.

With respect to the survival outcomes, the improvement in PFS of MATKIs failed to translate into overall survival benefits. In the perspective of trial design, this might be attributed to the confounding effects from bias of the subsequent treatments. This speculation was supported by the current subgroup analyses which showed that trials studying the maintenance $/ 2^{\text {nd }}$-line $/ 3^{\text {rd }}$-line settings have greater magnitudes of benefits compared with those studying the $1^{\text {st }}$-line settings. This evidence highlighted the advantages of using MATKIs and highlighted the need for balancing post-trial therapies in the future studies.

Since increased risk of hemoptysis in squamous cell carcinoma was documented during the early trials, bevacizumab is only indicated for non-squamous NSCLC [35]. Similarly, part of trials on MATKIs excluded patients with squamous cell carcinoma. Through subgroup analyses, we observed that these trials did not reveal any advantages of MATKI-containing regimens over MATKI-free regimens. However, significant differences were presented when pooling the studies that recruited all histological types (including squamous cell carcinoma). It at least suggested that non-squamous NSCLC might not the targeted subpopulation that benefits most from MATKIs. An exploratory analysis revealed an increased pooled incidence of hemorrhage based on two studies including all histological types, while the incidence was similar between the groups based on the other two studies including only non-squamous NSCLC (data not shown). Although our results did not provide direct evidences, they suggested that patients with squamous cell carcinoma might benefit more from MATKI but might be associated with increased risk for hemorrhage compared with non-squamous NSCLC. However, some recent reports argued that the risk of hemorrhage among squamous NSCLC when using anti-angiogenic agents was acceptable [36]. Therefore, the inclusion criteria, in terms of histology, require further discussion for future studies on MATKIs. Of course, direct comparison of histology associated hemorrhage risk should be proposed to clarify this issue.

This is the most up-to-date comprehensive analysis to compare the MATKI-containing to MATKI-free regimens, confirming the true efficacy of MATKIs. Nevertheless, there are several limitations. Firstly, the meta-analysis might suffer from significant clinical heterogeneity. There were various chemotherapeutic 


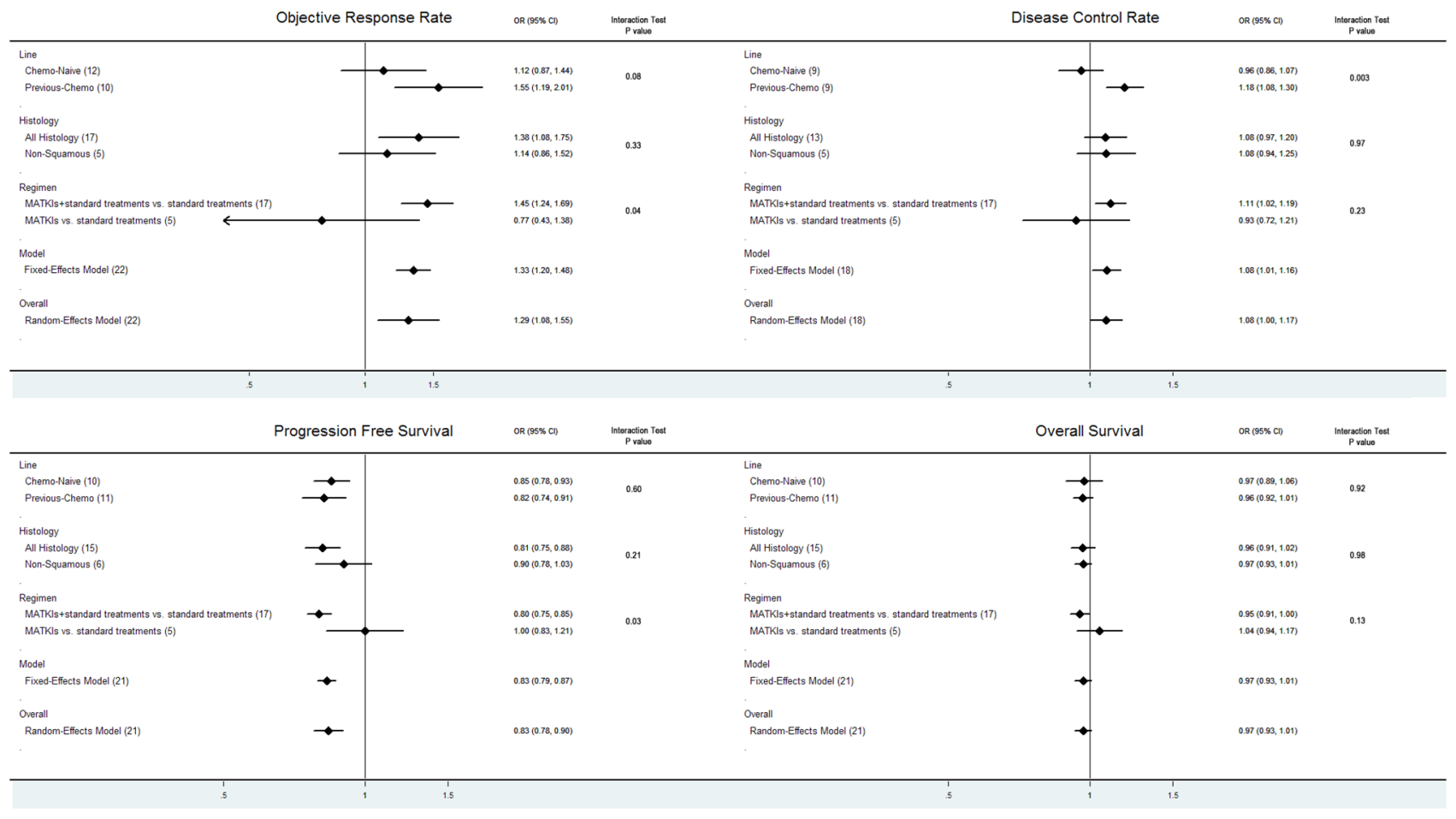

Figure 3. Subgroup analyses of each endpoint (number of studies or arms). A, ORR; B, DCR; C, PFS; D, OS. doi:10.1371/journal.pone.0109757.g003

regimens and patterns involved but we were unable to evaluate the respective effect of different MATKIs or treatment settings since current data were not sufficient to draw any solid conclusion for each subset. Secondly, only six agents were finally included in the analysis because studies on other agents were still ongoing (http:// clinicaltrials.gov). Thirdly, we were unable to conduct this analysis based on individual patient data, which could definitely provide more reliable information especially in subgroup analyses. To be sure, further studies are warranted to complete the information.

In conclusion, regimens consisting of multi-targeted antiangiogenic TKIs were superior to those without these agents in terms of ORR and PFS in patients with advanced NSGLC. However, no significant benefits in DCR or OS were observed. In addition, subgroup analyses provided us some hints to improve future studies and clinical application of MATKIs.

\section{References}

1. Jemal A, Siegel R, Xu J, Ward E. Cancer statistics, 2010. CA Cancer J Clin 2010; 60: 277-300.

2. National Comprehensive Cancer Network. NCGN Clinical Practice Guidelines in OncologyTM. Non-Small Cell Lung Cancer. V 2.2010. http://www.nccn. org/professionals/physician gls/PDF/nscl.pdf. Accessed April 8; 2010.

3. Ramalingam S, Belani C. Systemic chemotherapy for advanced non-small cell lung cancer: recent advances and future directions. Oncologist 2008; (Suppl. 1): 5-13.

4. Ferrara N, Gerber HP, LeCouter J. The biology of VEGF and its receptors. Nat Med 2003; 9: 669-76.

5. Carmeliet P, Jain RK. Angiogenesis in cancer and other diseases. Nature 2000; 407(6801): 249-57.

6. Sandler A, Gray R, Perry MC, Brahmer J, Schiller JH, et al. Paclitaxel carboplatin alone or with bevacizumab for non-smallcell lung cancer. N Engl J Med 2006; 355: 2542-2550.

7. Ivy SP, Wick JY, Kaufman BM. An overview of small molecule inhibitors of VEGFR signaling. Nat Rev Clin Oncol 2009; 6: 569-579.

8. Dearden S, Stevens J, Wu YL, Blowers D. Mutation incidence and coincidence in non small-cell lung cancer: meta-analyses by ethnicity and histology (mutMap). Ann Oncol 2013; 24(9): 2371-6.

\section{Supporting Information}

Table S1 Summary of Subgroup Analyses Results. (DOC)

\section{Checklist S1 PRISMA checklist.}

(PDF)

Flowchart S1 PRISMA flow chart.

(PDF)

\section{Author Contributions}

Conceived and designed the experiments: WHL XW SDH LZ. Performed the experiments: WHL XW YXZ SYK. Analyzed the data: WHL SDH WFF TQ. Contributed reagents/materials/analysis tools: WFF YH HYZ. Contributed to the writing of the manuscript: WHL XW YXZ.

9. Wozniak A. Challenges in the current antiangiogenic treatment paradigm for patients with non-small cell lung cancer. Crit Rev Oncol Hematol. 2012 May; 82(2): 200-12

10. Xiao YY, Zhan P, Yuan DM, Liu HB, Lv TF, et al. Chemotherapy plus multitargeted antiangiogenic tyrosine kinase inhibitors or chemotherapy alone in advanced NSCLC: a meta-analysis of randomized controlled trials. Eur J Clin Pharmacol 2013; 69(2): 151-9.

11. Jadad AR, Moore RA, Carroll D, Jenkinson C, Reynolds DJ, et al. Assessing the quality of reports of randomized clinical trials: is blinding necessary? Control Clin Trials 1996; 17: 1-12.

12. Egger M, Davey Smith G, Schneider M, Minder C. Bias in meta-analysis detected by a simple, graphical test. BMJ 1997; 315(7109): 629-34.

13. Begg CB, Mazumdar M. Operating characteristics of a rank correlation test for publication bias. Biometrics 1994; 50: 1088-1101.

14. Socinski MA, Scappaticci FA, Samant M, Kolb MM, Kozloff MF. Safety and efficacy of combining sunitinib with bevacizumab + paclitaxel/carboplatin in non-small cell lung cancer. J Thorac Oncol 2010; 5(3): 354-60.

15. Scagliotti GV, Krzakowski M, Szczesna A, Strausz J, Makhson A, et al Sunitinib plus erlotinib versus placebo plus erlotinib in patients with previously treated advanced non-small-cell lung cancer: a phase III trial. J Clin Oncol 2012; 30(17): 2070-8. 
16. Spigel DR, Burris HA 3rd, Greco FA, Shipley DL, Friedman EK, et al. Randomized, double-blind, placebo-controlled, phase II trial of sorafenib and erlotinib or erlotinib alone in previously treated advanced non-small-cell lung cancer. J Clin Oncol 2011; 29(18): 2582-9.

17. Scagliotti G, Novello S, von Pawel J, Reck M, Pereira JR, et al. Phase III study of carboplatin and paclitaxel alone or with sorafenib in advanced non-small-cell lung cancer. J Clin Oncol 2010; 28(11): 1835-42.

18. Paz-Ares LG, Biesma B, Heigener D, von Pawel J, Eisen T, et al. Phase III, randomized, double-blind, placebo-controlled trial of gemcitabine/cisplatin alone or with sorafenib for the first-line treatment of advanced, nonsquamous non-small-cell lung cancer. J Clin Oncol 2012; 30(25): 3084-92.

19. Wang Y, Wang L, Liu Y, Yu S, Zhang X, et al. Randomize trial of cisplatin plus gemcitabine with either sorafenib or placebo as first-line therapy for non-small cell lung cancer. [Article in Chinese] Zhongguo Fei Ai Za Zhi 2011; 14(3): 23944 .

20. Molina JR, Dy GK, Foster NR, Allen Ziegler KL, Adjei A, et al. A randomized phase II study of pemetrexed (PEM) with or without sorafenib (S) as second-line therapy in advanced non-small cell lung cancer (NSCLC) of nonsquamous histology: NCCTG N0626 study. Journal of Clinical Oncology 2011; 29 [Abstract 7513].

21. Goss GD, Arnold A, Shepherd FA, Dediu M, Ciuleanu TE, et al. Randomized, double-blind trial of carboplatin and paclitaxel with either daily oral cediranib or placebo in advanced non-small-cell lung cancer: NCIC clinical trials group BR24 study. J Clin Oncol 2010; 28(1): 49-55.

22. Dy GK, Mandrekar SJ, Nelson GD, Meyers JP, Adjei AA, et al. A randomized phase II study of gemcitabine and carboplatin with or without cediranib as firstline therapy in advanced non-small-cell lung cancer: North Central Cancer Treatment Group Study N0528. J Thorac Oncol 2013; 8(1): 79-88.

23. Laurie SA, Solomon BJ, Seymour L, Ellis PM, Goss GD, et al. A randomized double-blind trial of carboplatin plus paclitaxel (CP) with daily oral cediranib (CED), an inhibitor of vascular endothelial growth factor receptors, or placebo (PLA) in patients (pts) with previously untreated advanced non-small cell lung cancer (NSCLC): NCIC Clinical Trials Group study BR.29. J Clin Oncol 2012; 30 S:abstr 7511.

24. Heymach JV, Johnson BE, Prager D, Csada E, Roubec J, et al. Randomized, placebo-controlled phase II study of vandetanib plus docetaxel in previously treated non small-cell lung cancer. J Clin Oncol 2007; 25(27): 4270-7.

25. Herbst RS, Sun Y, Eberhardt WE, Germonpré P, Saijo N, et al. Vandetanib plus docetaxel versus docetaxel as second-line treatment for patients with advanced non-small-cell lung cancer (ZODIAC): a double-blind, randomised, phase 3 trial. Lancet Oncol 2010; 11(7): 619-26.

26. de Boer RH, Arrieta Ó, Yang CH, Gottfried M, Chan V, et al. Vandetanib plus pemetrexed for the second-line treatment of advanced non-small-cell lung cancer: a randomized, double-blind phase III trial. J Clin Oncol 2011; 29(8): $1067-74$.

27. Heymach JV, Paz-Ares L, De Braud F, Sebastian M, Stewart DJ, et al Randomized phase II study of vandetanib alone or with paclitaxel and carboplatin as first-line treatment for advanced non-small-cell lung cancer. J Glin Oncol 2008; 26(33): 5407-15.

28. Natale RB, Thongprasert S, Greco FA, Thomas M, Tsai CM, et al. Phase III trial of vandetanib compared with erlotinib in patients with previously treated advanced non-small-cell lung cancer. J Clin Oncol 2011; 29(8): 1059-66.

29. Natale RB, Bodkin D, Govindan R, Sleckman BG, Rizvi NA, et al. Vandetanib versus gefitinib in patients with advanced non-small-cell lung cancer: results from a two-part, double-blind, randomized phase ii study. J Clin Oncol 2009; 27(15): 2523-9.

30. Lee JS, Hirsh V, Park K, Qin S, Blajman CR, et al. Vandetanib Versus placebo in patients with advanced non-small-cell lung cancer after prior therapy with an epidermal growth factor receptor tyrosine kinase inhibitor: a randomized, double-blind phase III trial (ZEPHYR). J Clin Oncol 2012; 30(10): 1114-21.

31. Scagliotti GV, Vynnychenko I, Park K, Ichinose Y, Kubota K, et al. International, randomized, placebo-controlled, double-blind phase III study of motesanib plus carboplatin/paclitaxel in patients with advanced nonsquamous non-small-cell lung cancer: MONET1. J Clin Oncol 2012; 30(23): 2829-36.

32. Blumenschein GR Jr, Kabbinavar F, Menon H, Mok TS, Stephenson J, et al. A phase II, multicenter, open-label randomized study of motesanib or bevacizumab in combination with paclitaxel and carboplatin for advanced nonsquamous non-small-cell lung cancer. Ann Oncol 2011; 22(9): 2057-67.

33. Reck M, Kaiser R, Mellemgaard A, Douillard JY, Orlov S, et al. Docetaxel plus nintedanib versus docetaxel plus placebo in patients with previously treated nonsmall-cell lung cancer (LUME-Lung 1): a phase 3, double-blind, randomised controlled trial. Lancet Oncol 2014; 15(2): 143-55.

34. Cesca M, Bizzaro F, Zucchetti M, Giavazzi R. Tumor Delivery of Chemotherapy Combined with Inhibitors of Angiogenesis and Vascular Targeting Agents. Front Oncol 2013; 3: 259.

35. Johnson DH1, Fehrenbacher L, Novotny WF, Herbst RS, Nemunaitis JJ, et al. Randomized phase II trial comparing bevacizumab plus carboplatin and paclitaxel with carboplatin and paclitaxel alone in previously untreated locally advanced or metastatic non-small-cell lung cancer. J Clin Oncol 2004; 22(11): 2184-91.

36. Hellmann MD1, Chaft JE, Rusch V, Ginsberg MS, Finley DJ, et al. Risk of hemoptysis in patients with resected squamous cell and other high-risk lung cancers treated with adjuvant bevacizumab. Cancer Chemother Pharmacol 2013; 72(2): 453-61. 